\title{
Effect of Zishenpingchan Granule on Neurobehavioral Manifestations and the Activity and Gene Expression of Striatal Dopamine D1 and D2 Receptors of Rats with Levodopa-Induced Dyskinesias
}

\author{
Qing Ye, ${ }^{1}$ Xiao-Lei Yuan, ${ }^{1}$ Jie Zhou, ${ }^{1}$ Can-xing Yuan, ${ }^{1}$ and Xu-ming Yang ${ }^{2}$ \\ ${ }^{1}$ Department of Neurology, Longhua Hospital Affiliated to Shanghai University of Traditional Chinese Medicine, \\ Shanghai 200032, China \\ ${ }^{2}$ Department of Acupuncture Institute, Shanghai University of Traditional Chinese Medicine, Shanghai 201203, China
}

Correspondence should be addressed to Can-xing Yuan; ycanxing@hotmail.com and Xu-ming Yang; fslbest@126.com

Received 24 July 2014; Revised 10 October 2014; Accepted 17 October 2014; Published 12 November 2014

Academic Editor: Shan-Yu Su

Copyright (C) 2014 Qing Ye et al. This is an open access article distributed under the Creative Commons Attribution License, which permits unrestricted use, distribution, and reproduction in any medium, provided the original work is properly cited.

\begin{abstract}
This study was performed to observe the effects of Zishenpingchan granule on neurobehavioral manifestations and the activity and gene expression of striatal dopamine D1 and D2 receptors of rats with levodopa-induced dyskinesias (LID). We established normal control group, LID model group, and TCM intervention group. Each group received treatment for 4 weeks. Artificial neural network (ANN) was applied to excavate the main factor influencing variation in neurobehavioral manifestations of rats with LID. The results showed that overactivation in direct pathway mediated by dopamine D1 receptor and overinhibition in indirect pathway mediated by dopamine D2 receptor may be the main mechanism of LID. TCM increased the efficacy time of LD to ameliorate LID symptoms effectively mainly by upregulating dopamine D2 receptor gene expression.
\end{abstract}

\section{Introduction}

Parkinson's disease (PD) is a common chronic progressive neurodegenerative disorder in middle-aged and elderly people. The main pathological characteristic of PD is degeneration of dopaminergic neurons in the substantia nigra compact. At present, levodopa is still the gold standard for treating motor symptoms of PD, but more than 50\% of PD patients treated with levodopa develop levodopainduced dyskinesias in long-term therapy (generally over 5 years) $[1,2]$. The major clinical manifestations are chorea or athetoid involuntary movements, myotonia, or myoclonus. Torso and head-face are also involved. It has a serious impact on the life quality of PD patients. The development of LID is related to loss of dopaminergic neurons control in the substantia nigra-striatum and the pulsatile treatment of $\mathrm{LD}$, but the exact mechanism is not clear. Most consider that
LID is due to the imbalance between the direct and indirect pathways in basal ganglia and also due to dopamine receptor hypersensitivity $[3,4]$. Therefore, it is important to clarify the mechanism of LD inducing LID and find prevention and control measures, so as to improve the symptoms and the life quality of PD's advanced stage. This study mainly focused on the effect of Zishenpingchan granule on neurobehavioral manifestations and the activity and gene expression of striatal dopamine D1 and D2 receptors of rats with LID and provided theoretical and experimental basis for further study.

\section{Materials and Methods}

2.1. Drugs. Zishenpingchan granule is an effective Chinese herbal drug of the old Shanghai famous specialist of TCM Jianhua Hu. It is composed of Shudihuang $15 \mathrm{~g}$, Gouqi $15 \mathrm{~g}$, Sangjisheng $20 \mathrm{~g}$, Tianma $15 \mathrm{~g}$, Jiangcan $10 \mathrm{~g}$, Ezhu $15 \mathrm{~g}$, 
Baishaoyao 20 g, Tiannanxing 15 g, Quanxie 3 g, and Wugong $3 \mathrm{~g}$. And it was made into granule by Jiangyin Tianjiang Pharmaceutical Co., Ltd. (Lot. 0504312). Each bag weighs $4.8 \mathrm{~g}$ equivalent to $32 \mathrm{~g}$ of crude drug. We mixed 4 bags of Zishenpingchan granule (equivalent to adult dosage of a day, containing $128 \mathrm{~g}$ of crude drug) and dissolved it in $100 \mathrm{~mL}$ normal saline. Each $1 \mathrm{~mL}$ contains $1.28 \mathrm{~g}$ crude drugs. LD powder (Lot. SLD6382) and benserazide powder (Lot. SL06492) were provided by Sigma-Aldrich (Shanghai) Co., Ltd., America.

2.2. Animals. Male SD rats (SCXK 2003-0002), weighing 180-220 g each, were purchased from Shanghai Sippr BK Laboratory Animals Ltd.

2.3. Instruments and Reagents. Apomorphine (APO), 6-hydroxydopamine (6-OHDA), SCH23390, and Spiperone were obtained from Sigma-Aldrich (Shanghai) Co., Ltd., USA. 3HSCH23390 (69.8 Ci/mmol) and 3H-Spiperone (14.1 Ci/mmol) were from Amersham Co., Ltd., USA. TaKaRa reserve transcription reagent and PCR reactions kit were provided by TaKaRa Biotechnology (Dalian) Co., Ltd., while DEPC was from SBS Company. Rat brain stereotaxis instrument (T0w-3A) was from the Second Military Medical University. Ultracentrifuge (LE80K) was from Beckman Company. Liquid scintillation luminescence numeration (Wallacl450) was from PerkinElmer Inc., USA. IQS Multicolor Real-Time PCR Detection System was from BIO-RAD, USA, and nucleic acid protein detector was from Eppendorf, Germany.

2.4. Preparation of PD Rat Model [5]. Adult Sprague-Dawley (SD) rats were anesthetized by $1 \%$ pentobarbital before they were fixed on the rat stereotaxis instrument referring to the brain stereotaxic atlas made by Bao and Shu [6] and we determined the 3D coordinate of right SNC and VTA. (1) SNC: it was behind the anterior fontanelle $4.8 \mathrm{~mm}$, on the right side of the sagittal suture $2.0 \mathrm{~mm}$, and under the dura $8.0 \mathrm{~mm}$. (2) VTA: it was behind the anterior fontanelle $4.8 \mathrm{~mm}$, on the right side of the sagittal suture $1.2 \mathrm{~mm}$, and under the dura $8.2 \mathrm{~mm}$. We punched holes by using dental drill and injected 6-OHDA $6 \mu \mathrm{g}$ into the two coordinates (soluble in water mass fraction of saline $0.2 \%$ ascorbic acid, concentration of $2 \mu \mathrm{g} / \mu$ ) with injection speed $1 \mu \mathrm{L} / \mathrm{min}$ and needle retaining time of $10 \mathrm{~min}$. After operation, we packed skull hole with the gelatin sponge, sutured the skin incision, and injected gentamicin and then put the rats back to the rearing cage after they had woken up. Behavioral test began at 2 weeks after operation, using intraperitoneal injection of Apomorphine $(0.5 \mathrm{mg} / \mathrm{kg})$, when rats were constantly turned to the left and the rotational number $>210 \mathrm{r} / 30 \mathrm{~min}$ is regarded as a successful model of PD. Normal control group rat model preparation: and then we injected normal saline into right SNC and VTA, respectively, using the method described above (only containing $0.2 \%$ ascorbic acid). Processing method is in the same place after the operation. The experiment was approved by the Experimental Animal Ethics Committee of Shanghai University of Traditional Chinese Medicine in China (Approval number 09047).
2.5. Preparation of LID Rat Model. PD rats with successful modeling were produced by intraperitoneally injecting LD $(10 \mathrm{mg} / \mathrm{kg})$ and benserazide $(2.5 \mathrm{mg} / \mathrm{mL}$, dissolved in sterile normal saline with $0.05 \%$ ethanol and $0.05 \%$ ascorbic acid) twice a day (10 mg/kg per day) for 4 weeks. And we screened out the LID model based on presence of AIM including stereotyped act and contralateral rotation [7] (specific provisions of AIM score > 20 as a successful model of the LID).

\subsection{Determination of Neurobehavioral Manifestations}

2.6.1. Determination of AIM Scores. AIM was measured in each group once a week as described by Cao et al. [8]. The assessment of rats was done every 30 min immediately after injection of LD and benserazide and continued for $120 \mathrm{~min}$. There were five sets of data, and the sum of all the data was the final evaluation results. We divided AMI score into 4 parts (fore leg, oral-facial region, axial, and movement) for evaluation. Each part was divided into 5 levels on the scale of zero to 4: zero for none; 1 for occasionally; 2 for frequently occurring; 3 for persistency but stimulation can make it stop; 4 for persistency and stimulation cannot make it stop. Theoretically, the highest AMI score of a rat after drug taken once is 64 .

2.6.2. Peak Dose Rotation [9]. On the 7th, 14th, 21st, and 28th mornings during the treatment, neurobehavioral manifestations and AMI score of each group were observed. After injection of $\mathrm{LD}$, we recorded rotation every $5 \mathrm{~min}$. The largest number of rotation was the peak dose rotation.

2.6.3. Efficacy Time of $\operatorname{LD}[10,11]$. The observation of neurobehavioral manifestations was performed once a week. We recorded the contralateral rotation laps every $5 \mathrm{~min}$ after injection of LD within 2 hours. Then we calculated the total laps of rotation and obtained the average number of rotation laps every $5 \mathrm{~min}$ within 2 hours. And we took the time between the first $5 \mathrm{~min}$ the number of rotation laps increased to the half of the average number and the first $5 \mathrm{~min}$ the number decreased to the half of average after injection of LD as the efficacy time of LD.

2.7. Grouping and Drug Administration of Experimental Animals. Totally $22 \mathrm{PD}$ rats of 42 rats were successfully modeled according to the determination of neurobehavioral manifestations. After 4 weeks of LD treatment, 16 successful LID rats were screened out and divided into LID group and TCM group, 8 rats each. Another 8 rats served as control group. LID group continued to get injection of LD and benserazide $(10 \mathrm{mg} / \mathrm{kg}$, twice a day). TCM group rats were given TCM $(9 \mathrm{~mL} / \mathrm{kg}$, once a day, i.g.) on the basis of LID group. And rats in the control group were injected and given intragastric administration of NS for 4 weeks.

2.8. Rat Brain Tissue RNA Isolation. Rats were anesthetized by $1 \%$ sodium pentobarbital at 2 hours after the last administration. After decapitation, brain tissues were stripped in an ice bath and the bilateral midbrain nigra and caudate putamen brain tissue were accurately cut and transferred 
to the homogenizer, respectively. They were kept with $1 \mathrm{~mL}$ Trizol at room temperature for 5-10 minutes. Then they were transferred to doff tubes, added to $0.2 \mathrm{~mL}$ chloroform, and vibrated for $15 \mathrm{~min}$. After 3 minutes' standing at $4^{\circ} \mathrm{C}$ room temperature, $4^{\circ} \mathrm{C} 12000 \mathrm{~g}$ (15000 rpm) for $15 \mathrm{~min}$, supernatant was transferred to another doff tube and added to $0.5 \mathrm{~mL}$ isopropyl alcohol, still standing at $4^{\circ} \mathrm{C}$ for $15 \mathrm{~min}, 4^{\circ} \mathrm{C} 12000 \mathrm{~g}$ $(15000 \mathrm{rpm})$ for $10 \mathrm{~min}$. After removal of supernatant, we added $1 \mathrm{~mL} 95 \%$ ethanol for washing the precipitation, $4^{\circ} \mathrm{C}$ $8000 \mathrm{~g}(10000 \mathrm{rpm})$ for $5 \mathrm{~min}$. Then we dried the deposition and added DEPC to dissolve, kept at $20^{\circ} \mathrm{C}$.

2.9. Reverse Transcription. FQ-PCR was used with the primers (10D each pair): D1 mRNA: F (GCTATGCTGACTGGGCTGAC); R (TTTCAGGGATGCTGCCTCT); D2 mRNA: F (CACTCAGATGCTTGCCATTGTTC); R (GTGGGATGTTGCAATCACAGTGTA). Reaction conditions were as follows: at $37^{\circ} \mathrm{C}$ for $15 \mathrm{~min}$ to the reverse transcription reaction and at $85^{\circ} \mathrm{C}$ for $5 \mathrm{sec}$ to the inactivation of reverse transcriptase reaction.

2.10. FQ-PCR Reaction Conditions. We measured the relative content of each mRNA by using quantitative PCR: $95^{\circ} \mathrm{C}$, $0.05 \mathrm{~min} \rightarrow T_{m}: 0.2 \mathrm{~min} \rightarrow 72^{\circ} \mathrm{C}, 0.1 \mathrm{~min} \rightarrow 72^{\circ} \mathrm{C}$, and $5 \mathrm{~min}$ $\left(T_{m}\right.$ : B-action-STR $61.6^{\circ} \mathrm{C}$, B-action SN $61.4^{\circ} \mathrm{C}, \mathrm{D} 157.0^{\circ} \mathrm{C}$, and D2 $60.0^{\circ} \mathrm{C}$ ). According to the FQ-PCR indirect quantitative formula, the average relative content $\%=2$ average $-\Delta \Delta \mathrm{Ct}, \Delta \Delta \mathrm{Ct}=\Delta \mathrm{Ct}$ sample $-\Delta \mathrm{Ct}$ control, $\Delta \mathrm{Ct}$ sample $=$ $\Delta \mathrm{Ct}$ sample $-\Delta \mathrm{Ct}$ internal control.

2.11. Preparation of Membrane Proteins. We prepared the membrane protein as described by Zhang et al. [12]. Rats were anesthetized by $1 \%$ sodium pentobarbital at 2 hours after the last administration. After decapitation, brain tissues were stripped on ice and the bilateral midbrain nigra and caudate putamen brain tissue were accurately cut, respectively. And we added precooling centrifugal buffer of $4^{\circ} \mathrm{C}$ and made it into homogenate in an ice bath. The precipitate was diluted in 1-2 $\mathrm{mL}$ trishydrochloric acid buffer ( $\mathrm{pH}$ 7.4) and fully suspended to membrane protein suspension. The membrane protein level was measured by Coomassie brilliant blue method and adjusted to $1 \mathrm{~g} / \mathrm{L}$.

2.12. Determination of Dopamine Receptor Activity. Using the double compound tubes method (each sample was divided into 9 different concentrations of reaction tube, repeat test twice), we added marked ligands whose concentration was increasing by multiple (specific binding, 10, 20, 40, 60, 80 , and $100 \mu \mathrm{L}$; nonspecific binding 20, 60, and $100 \mu \mathrm{L}$ ), nonmarked ligands (nonspecific binding, $100 \mu \mathrm{L}$ ), and $0.2 \mathrm{~mL}$ quantitative membrane proteins solution got into the reaction tube. We also added buffer into each tube to make the total reaction volume $0.4 \mathrm{~mL}$. After $15 \mathrm{~min}$ incubation in $37^{\circ} \mathrm{C}$ bath, the reaction stopped in an ice bath. We collected long cell sample on fiberglass filter paper by using a collector and dried it in a drier of $80^{\circ} \mathrm{C}$. Then we cut off the sample and measured its radioactivity by using $2000 \mathrm{CA} / \mathrm{LL}$ liquid scintillation counter. It was corrected in sample application with blank filter paper. And we used receptor data package (documented by Shanghai Second Medical University) to calculate specific binding count of each point. $B_{\max }$ (fmol.mg) and $\mathrm{KD}(\mathrm{nmol} \cdot \mathrm{L})$ of dopamine receptors were calculated according to Scanchard formulas.

2.13. Statistical Analysis. All results are expressed as the means \pm SD. The data were analyzed with SPSS 18.0 software.

2.13.1. Statistical Analysis of Neurobehavioral Manifestations. Firstly, Mauchly's test of sphericity was used to judge whether there were relations among the repeated measures data. If any $(P<0.05)$, multivariate ANOVA should be taken next, or Greenhouse-Geisser corrected results should be taken. Treated effect could be evaluated by estimating betweensubject variance. Repeated measurement effect or its interactive effect with treated group could be evaluated by estimating within-subject variance. The method of Bonferroni should be used to do pairwise comparisons of the repeated measures data at different measurement time points of each treated group. With multivariate ANOVA, data in different treated group at each measurement time point could be compared pairwise. Differences were considered significant at $P<0.05$.

2.13.2. Statistical Analysis of Dopamine Receptors Determination. Comparison between two groups was done with independent sample $t$-test and comparison among groups was done with one-way ANOVA analysis. Differences were considered significant at $P<0.05$. Statistical results were graphed with Excel and SPSS 18.0 software.

2.14. Data Mining Based on an Artificial Neural Network $B P(B P-A N N)$. BP-ANN could approximate any nonlinear curve [13]. To identify which factors were associated with the AIM score change and the peak dose rotation change, the network was given a set of inputs and corresponding outputs and determined the parameters of each neuron by analyzing the relationship between the input and output. We selected mRNA of lesion D1 receptor, mRNA of lesion D2 receptor, $B_{\max }$ of lesion $\mathrm{D} 1$ receptor, $B_{\max }$ of lesion $\mathrm{D} 2$ receptor, $\mathrm{KD}$ of lesion $\mathrm{D} 1$ receptor, and $\mathrm{KD}$ of lesion $\mathrm{D} 2$ receptor as parameters of the input. Parameters of the output were difference of AIM score and difference of peak dose rotation before and after treatment. After building the model, we could get a stable prediction and judgment model through training and learning of the input samples (as shown in Figure 1).

\section{Results}

\subsection{AIM Manifestations of LID Rats. See Figure 2.}

3.2. Comparison of Neurobehavioral Manifestations (AIM Score) before and after Dosing between Different Groups (Shown in Tables 1 and 2 and Figure 3). ANOVA results of repeated measures data showed that the differences between the groups were statistically significant $(P<0.01)$ (as shown in Table 1), which meant AIM scores were different under different treatment conditions. Time factor was statistically 


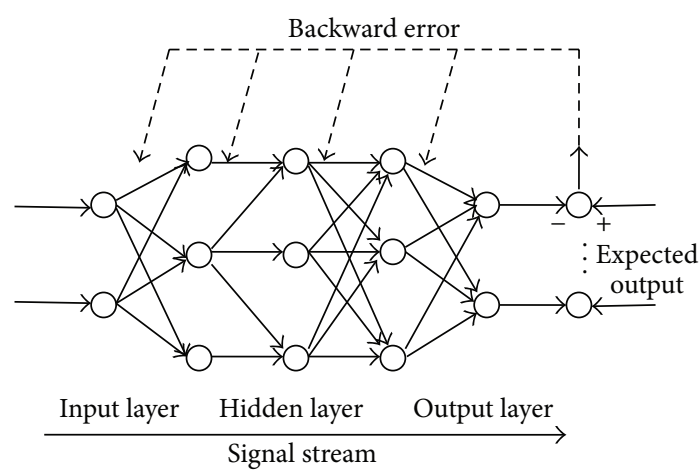

Figure 1: Mathematical description of BP-ANN.

TABLE 1: ANOVA results of repeated measures data of AIM score.

\begin{tabular}{lccccc}
\hline Source of variation & SS & DF & MS & $F$ & $P$ \\
\hline Variation within group & & & & & \\
$\quad$ Time & 1008.33 & 2.64 & 382.04 & 13.93 & $<0.01$ \\
Time group & 2182.68 & 2.64 & 826.98 & 30.16 & $<0.01$ \\
$\quad$ Error & 1013.40 & 36.95 & 27.43 & - & - \\
Variation between groups & & & & & \\
$\quad$ Group & 2633.51 & 1 & 2633.51 & 26.20 & $<0.01$ \\
$\quad$ Error & 1407.48 & 14 & 100.53 & - & - \\
\hline
\end{tabular}

significant $(P<0.01)$, which meant the measurement (AIM score) had a tendency to change over time. The interaction between group and time had obvious statistical significance $(P<0.01)$, which indicated AIM scores at each time point varied with treatment.

According to the results of multivariate ANOVA, on the 14th, 21st, and 28th days after treatment, the AIM score in LID group increased progressively $(P<0.01)$ compared with that before treatment (0th day) (as shown in Table 2 and Figure 3 ). On the 14th day, the AIM score of TCM group increased more than that before treatment $(P<0.05)$. After the 14 th day, AIM score decreased progressively and was significantly lower than that before treatment on the 28th day. Since the 14th day, the AIM score of TCM group was lower than that of LID group $(P<0.05)$, but there was no statistical significance between these two groups on the 7 th day.

3.3. Comparison of Neurobehavioral Manifestations (Peak Dose Rotation) before and after Dosing between Different Groups (Shown in Tables 3 and 4 and Figure 4). ANOVA results of repeated measures data showed the difference between the groups was statistically significant $(P<0.01)$ (as shown in Table 3), which meant peak dose rotation was different under different treatment conditions. Time factor was statistically significant $(P<0.01)$, which meant the measurement (peak dose rotation) had a tendency to change over time. The interaction between group and time had obvious statistical significance $(P<0.01)$, which indicated peak dose rotation at each time point varied with treatment.

According to the results of multivariate ANOVA (as shown in Table 4 and Figure 4), on the 7th, 14th, 21st, and 28th days after treatment, the peak dose rotation in LID group increased progressively $(P<0.05)$ compared with that before treatment (0th day). Since the 14th day, the rotation score of TCM group increased progressively $(P<0.01)$. The rotation score of TCM group was lower than that of LID group since the 14 th day $(P<0.01)$.

3.4. Comparison of Neurobehavioral Manifestations (LD Efficacy Time) before and after Dosing between Different Groups (Shown in Tables 5 and 6 and Figure 5). ANOVA results of repeated measures data showed the difference between the groups was statistically significant $(P<0.01)$ (as shown in Table 5), which meant LD efficacy time was different under different treatment conditions. Time factor was statistically significant $(P<0.01)$, which meant the measurement (LD efficacy time) had a tendency to change over time. The interaction between group and time had obvious statistical significance $(P<0.01)$, which indicated LD efficacy time at each time point varied with treatment.

According to the results of multivariate ANOVA (as shown in Table 6 and Figure 5), the LD efficacy time in LID group declined $(P<0.05)$ with the treatment time prolonged, compared with that before treatment (0th day). On the 21st day and 28th day, the efficacy time in TCM group was significantly higher than that before treatment $(P<0.01)$. But there was no obvious downward trend in TCM group. The LD efficacy time in TCM group was significantly higher than that in LID model group $(P<0.01)$ on the 21 st day and 28th day.

\subsection{Comparison of Dopamine Receptors Activity in Bilateral Caudate Putamen among Different Groups}

3.5.1. Comparison of Dopamine D1 Receptor Activity in Bilateral Caudate Putamen among Different Groups (Shown in Table 7). The $B_{\max }$ level of lesion side increased significantly $(P<0.01)$ in the LID group and the TCM group in comparison to that in the control group, and the LID group had the biggest rise. The KD level of lesion side reduced obviously $(P<0.05)$ in the LID group and the TCM group comparing to that in the control group. The $B_{\max }$ level decreased and the KD level increased $(P<0.01)$ in the TCM group compared to those in the LID group. Comparing to the control group, the $B_{\text {max }}$ level of normal side increased and KD level decreased in the LID group $(P<0.01)$. The $B_{\max }$ of normal side in the TCM group increased $(P<0.01)$, and other indexes had no evident differences. The $B_{\max }$ levels of lesion side were higher and the KD levels were lower than those of normal side in the LID group and the TCM group $(P<0.01)$.

3.5.2. Comparison of Dopamine D2 Receptor Activity in Bilateral Caudate Putamen among Different Groups (Shown in Table 8 ). The $B_{\max }$ level of lesion side decreased significantly $(P<0.01)$ in the LID group and the TCM group in comparison to that in the control group, and the LID group had the biggest falls $(P<0.01)$. The KD level of lesion side significantly elevated $(P<0.01)$ in the LID group comparing to that in the control group. The $B_{\max }$ level increased and 


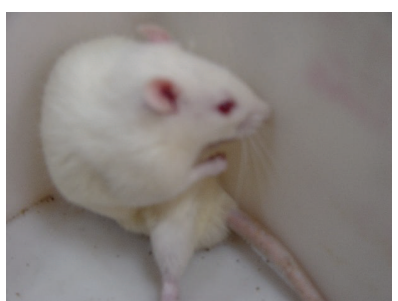

(a)

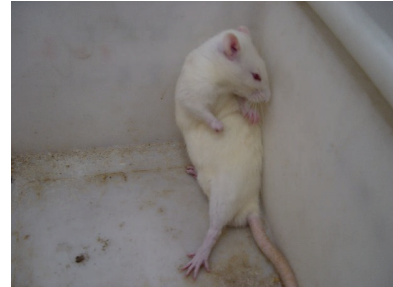

(b)

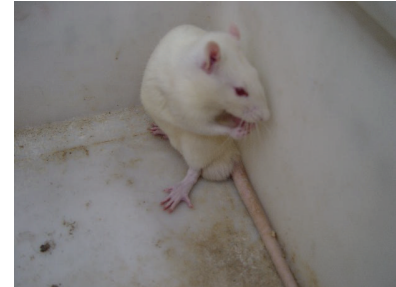

(c)

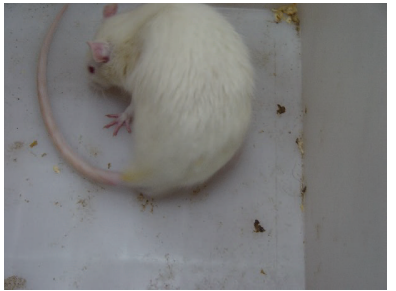

(d)

FIgURE 2: AIM manifestations of LID rats. (a) Limb movement disorder. (b) Axiality movement. (c) Chewing. (d) Contralateral rotation.

TABLE 2: Comparison of AIM score before and after dosing between different groups.

\begin{tabular}{|c|c|c|c|c|c|c|}
\hline \multirow{2}{*}{ Group } & \multirow{2}{*}{$n$} & \multicolumn{5}{|c|}{ Time point } \\
\hline & & 0th day & 7th day & 14th day & 21st day & 28th day \\
\hline LID & 8 & $34.63 \pm 2.97$ & $38.75 \pm 3.11$ & $48.50 \pm 9.55^{* *}$ & $51.88 \pm 6.60^{* *}$ & $53.88 \pm 5.06^{* *}$ \\
\hline TCM & 8 & $33.13 \pm 5.87$ & $38.13 \pm 9.05$ & $40.50 \pm 6.19^{\triangle}$ & $31.50 \pm 2.45^{\triangle \Delta}$ & $27.00 \pm 2.56^{\triangle \triangle}$ \\
\hline
\end{tabular}

AIM score ${ }^{*} P<0.05,{ }^{* *} P<0.01$ compared with 0 Day; AIM score ${ }^{\triangle} P<0.05,{ }^{\triangle} \triangle P<0.01$ compared with LID group.

TABLE 3: ANOVA results of repeated measures data of peak dose rotation.

\begin{tabular}{lccccc}
\hline Source of variation & SS & DF & MS & $F$ & $P$ \\
\hline Variation within group & & & & & \\
$\quad$ Time & 18801.88 & 1.74 & 10814.96 & 132.02 & $<0.01$ \\
Time.group & 2185.83 & 1.74 & 1257.30 & 15.35 & $<0.01$ \\
$\quad$ Error & 1933.90 & 24.34 & 81.92 & - & - \\
Variation between groups & & & & & \\
$\quad$ Group & 2070.61 & 1 & 2070.61 & 14.16 & $<0.01$ \\
Error & 2047.48 & 14 & 146.25 & - & - \\
\hline
\end{tabular}

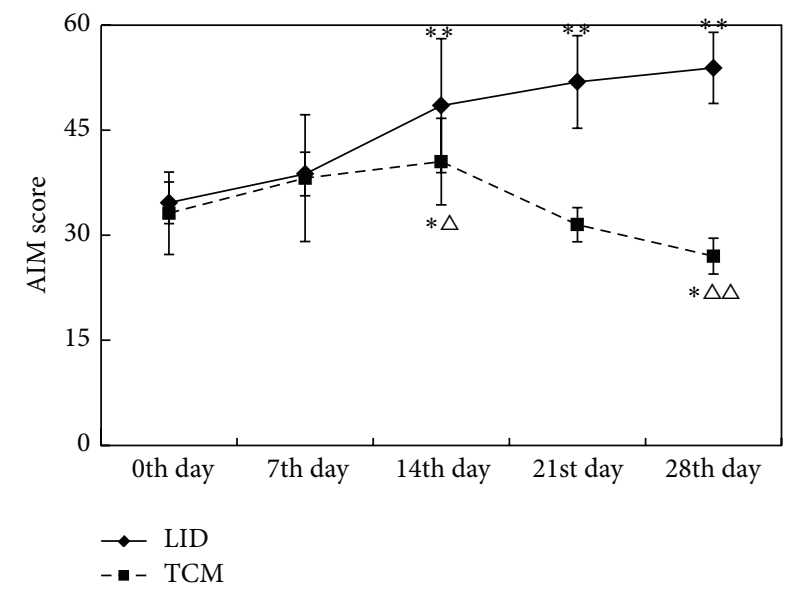

FIGURE 3: Comparison of AIM score before and after dosing between different groups. (AIM score ${ }^{*} P<0.05,{ }^{* *} P<0.01 \mathrm{com}-$ pared with 0th day; AIM score ${ }^{\triangle} P<0.05,{ }^{\triangle} P$ P 0.01 compared with LID group.)

the KD level reduced $(P<0.01)$ in the TCM group compared to those in the LID group $(P<0.01)$. Comparing to the control group, the $B_{\max }$ level of normal side decreased and KD level increased in the LID group $(P<0.05)$, and other

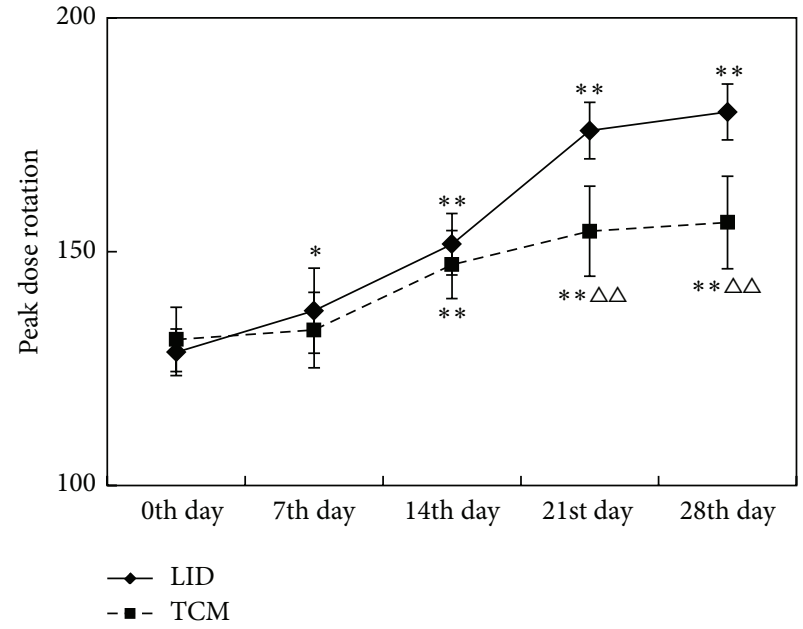

Figure 4: Comparison of peak dose rotation between different groups. (Peak dose rotation ${ }^{*} P<0.05,{ }^{* *} P<0.01$ compared with 0th day; peak dose rotation ${ }^{\triangle} P<0.05,{ }^{\triangle} \triangle P<0.01$ compared with LID group.)

indexes had no evident differences. The $B_{\max }$ levels of normal side were lower and the KD levels were higher than those of lesion side in the LID group $(P<0.01)$. And the $B_{\max }$ of lesion side was lower than that of normal side in the TCM group $(P<0.01)$.

3.6. Comparison of Gene Expression of Dopamine Receptors in Bilateral Caudate Putamen among Different Groups (Shown in Table 9 and Figures 6 and 7)

3.6.1. Comparison of $m R N A$ Expression of Dopamine D1 Receptor in Bilateral Caudate Putamen among Different Groups. The mRNA expression of dopamine D1 receptor of lesion side increased significantly $(P<0.05)$ in the LID group and 
TABLE 4: Comparison of peak dose rotation between different groups.

\begin{tabular}{|c|c|c|c|c|c|c|}
\hline \multirow{2}{*}{ Group } & \multirow{2}{*}{$n$} & \multicolumn{5}{|c|}{ Time point } \\
\hline & & 0th day & 7th day & 14 th day & 21st day & 28th day \\
\hline LID & 8 & $128.50 \pm 4.96$ & $137.38 \pm 9.09^{*}$ & $151.63 \pm 6.57^{* *}$ & $175.88 \pm 6.03^{* *}$ & $179.88 \pm 5.99^{* *}$ \\
\hline TCM & 8 & $131.25 \pm 6.90$ & $133.25 \pm 8.05$ & $147.25 \pm 7.27^{* *}$ & $154.38 \pm 9.61^{* * \Delta \Delta}$ & $156.25 \pm 9.87^{* * \Delta \triangle}$ \\
\hline
\end{tabular}

Peak dose rotation ${ }^{*} P<0.05,{ }^{* *} P<0.01$ compared with 0 Day; peak dose rotation ${ }^{\triangle} P<0.05,{ }^{\triangle} \triangle P<0.01$ compared with LID group.

TABLE 5: ANOVA results of repeated measures data of LD efficacy time.

\begin{tabular}{lccccc}
\hline Source of variation & SS & DF & MS & $F$ & $P$ \\
\hline Variation within group & & & & & \\
$\quad$ Time & 1349.83 & 4 & 337.46 & 8.04 & $<0.01$ \\
Time group & 696.33 & 4 & 174.08 & 4.15 & $<0.01$ \\
$\quad$ Error & 2349.85 & 56 & 41.96 & - & - \\
$\quad$ Variation among groups & & & & & \\
$\quad$ Group & 1073.11 & 1 & 1073.11 & 55.28 & $<0.01$ \\
$\quad$ Error & 271.78 & 14 & 19.41 & - & - \\
\hline
\end{tabular}

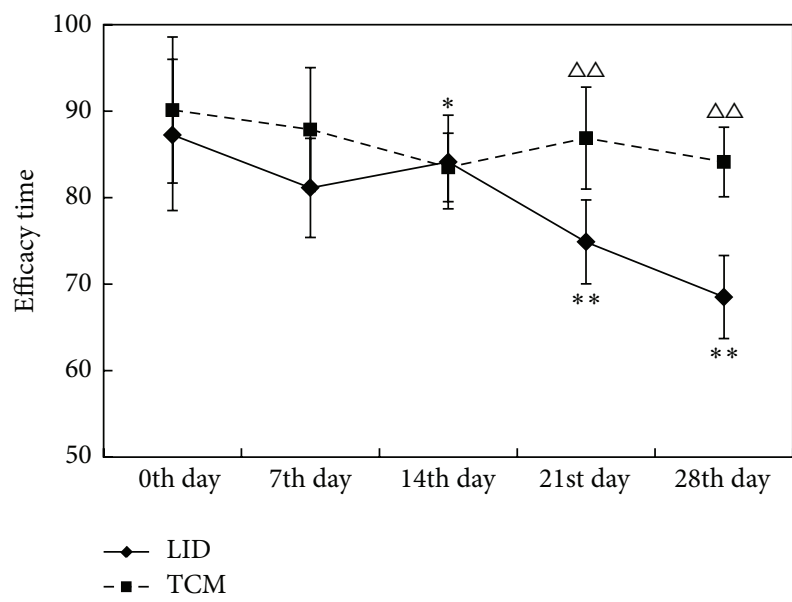

FIGURE 5: Comparison of LD efficacy time between different groups. (Peak dose rotation ${ }^{*} P<0.05,{ }^{* *} P<0.01$ compared with 0 th day; peak dose rotation ${ }^{\triangle} P<0.05,{ }^{\triangle}{ }^{\triangle} P<0.01$ compared with LID group.)

the TCM group in comparison to that in the control group, and the LID group had the biggest rise. The mRNA expression reduced $(P<0.01)$ in the TCM group comparing to that in the LID group. Comparing to the control group, the mRNA of normal side showed a significant rise in the LID group and the TCM group $(P<0.05)$, and others had no evident differences. The expression of lesion side was higher than that of normal side in the LID group $(P<0.05)$.

3.6.2. Comparison of mRNA Expression of Dopamine D2 Receptor in Bilateral Caudate Putamen among Different Groups. The mRNA expression of dopamine D2 receptor of lesion side declined obviously $(P<0.05)$ in the LID group and the TCM group in comparison to that in the control

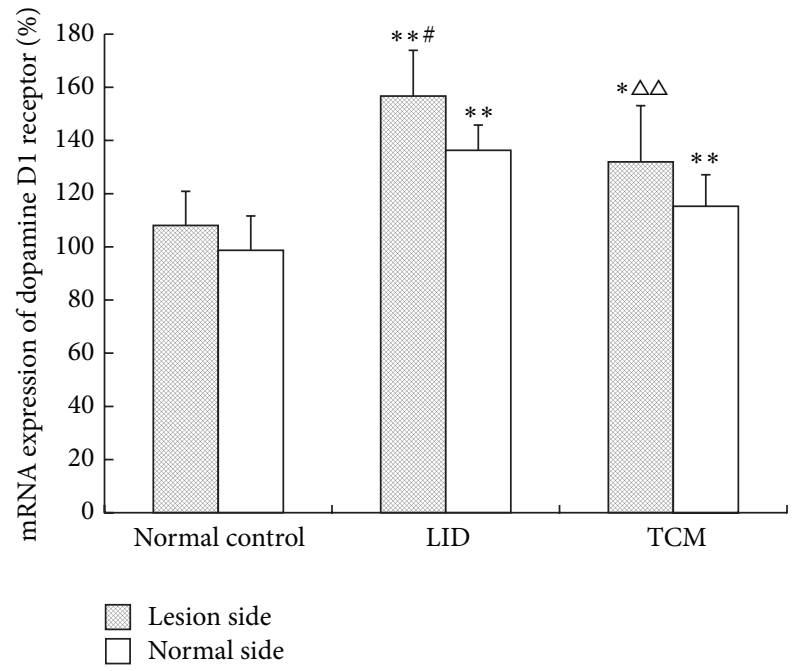

FIGURE 6: Comparison of the relative quantitative mRNA expression of dopamine D1 receptor in bilateral caudate putamen among different groups.

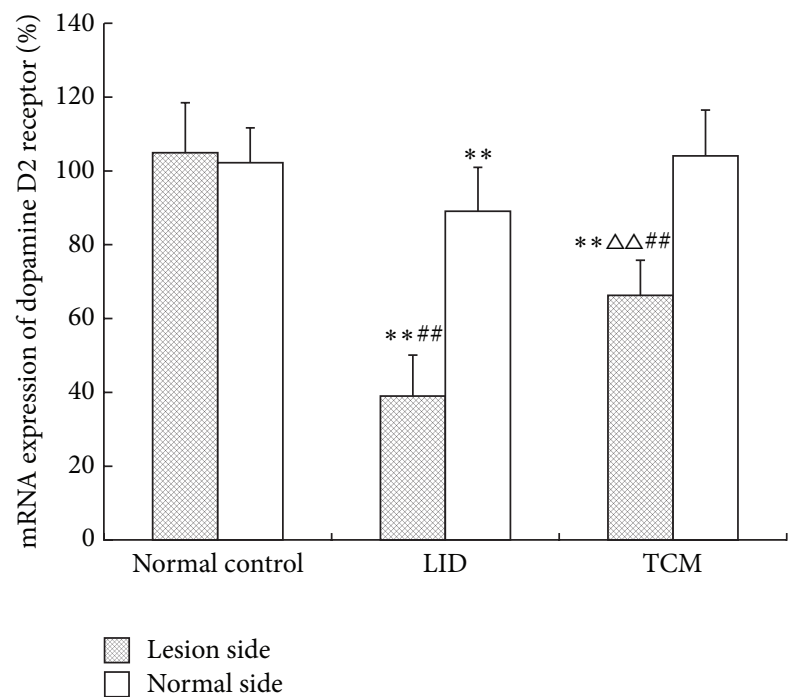

FIGURE 7: Comparison of the relative quantitative mRNA expression of dopamine D2 receptor in bilateral caudate putamen among different groups. $\left({ }^{*} P<0.05,{ }^{* *} P<0.01\right.$ compared with normal control group; ${ }^{\triangle} P<0.05,{ }^{\triangle} P<0.01$ compared with LID group; ${ }^{\#} P<0.05,{ }^{\# \#} P<0.01$ versus normal side.) 
TABLE 6: Comparison of LD efficacy time between different groups.

\begin{tabular}{|c|c|c|c|c|c|c|}
\hline \multirow{2}{*}{ Group } & \multirow{2}{*}{$n$} & \multicolumn{5}{|c|}{ Time point } \\
\hline & & 0th day & 7th day & 14th day & 21st day & 28th day \\
\hline LID & 8 & $82.75 \pm 8.75$ & $81.13 \pm 5.74$ & $84.13 \pm 5.41$ & $74.88 \pm 4.85^{* *}$ & $68.50 \pm 4.81^{* *}$ \\
\hline TCM & 8 & $90.13 \pm 8.44$ & $87.88 \pm 7.16$ & $83.50 \pm 3.96^{*}$ & $86.38 \pm 5.89^{\triangle \Delta}$ & $84.13 \pm 4.02^{\triangle \Delta}$ \\
\hline
\end{tabular}

Peak dose rotation ${ }^{*} P<0.05,{ }^{* *} P<0.01$ compared with 0 Day; peak dose rotation ${ }^{\triangle} P<0.05,{ }^{\triangle} P<0.01$ compared with LID group.

TABLE 7: Comparison of dopamine D1 receptor activity in bilateral caudate putamen among different groups $(\bar{x} \pm s)$.

\begin{tabular}{|c|c|c|c|c|c|}
\hline \multirow{2}{*}{ Group } & \multirow{2}{*}{$n$} & \multicolumn{2}{|c|}{$B_{\max }(\mathrm{fmol} / \mathrm{mg})$} & \multicolumn{2}{|c|}{$\mathrm{KD}(\mathrm{nmol} / \mathrm{L})$} \\
\hline & & Lesion side & Normal side & Lesion side & Normal side \\
\hline Normal control & 8 & $1118.99 \pm 86.84$ & $1075.22 \pm 90.92$ & $2.499 \pm 0.289$ & $2.554 \pm 0.216$ \\
\hline LID & 8 & $1653.63 \pm 59.88^{* * \# \#}$ & $1440.80 \pm 55.37^{* *}$ & $0.730 \pm 0.035^{* * \# \#}$ & $1.858 \pm 0.126^{* *}$ \\
\hline TCM & 8 & $1390.97 \pm 52.40^{* * \triangle \triangle \# \#}$ & $1306.00 \pm 51.64^{* *}$ & $1.342 \pm 0.063^{* * \triangle \triangle \# \#}$ & $2.485 \pm 0.197$ \\
\hline
\end{tabular}

${ }^{*} P<0.05,{ }^{* *} P<0.01$ compared with normal control group; ${ }^{\triangle} P<0.05,{ }^{\triangle} P<0.01$ compared with LID group; ${ }^{\#} P<0.05$, ${ }^{\# \#} P<0.01$ compared with normal side.

TABLE 8: Comparison of effect of TCM on dopamine D2 receptor activity in bilateral caudate putamen among different groups $(\bar{x} \pm s)$.

\begin{tabular}{lccccc}
\hline \multirow{2}{*}{ Group } & $n$ & \multicolumn{2}{c}{$B_{\max }(\mathrm{fmol} / \mathrm{mg})$} & \multicolumn{2}{c}{ KD $(\mathrm{nmol} / \mathrm{L})$} \\
& & Lesion side & Normal side & Lesion side & Normal side \\
\hline Normal control & 8 & $1284.87 \pm 155.54$ & $1246.71 \pm 124.45$ & $1.021 \pm 0.107$ & $0.996 \pm 0.120$ \\
LID & 8 & $533.16 \pm 44.14^{* * \# \#}$ & $1075.54 \pm 77.00^{*}$ & $1.587 \pm 0.050^{* * \# \#}$ & $1.204 \pm 0.075^{* *}$ \\
TCM & 8 & $771.85 \pm 103.00^{* * \Delta \triangle \# \#}$ & $1194.03 \pm 141.67$ & $1.067 \pm 0.070^{\triangle \Delta}$ & $1.097 \pm 0.124$ \\
\hline
\end{tabular}

${ }^{*} P<0.05,{ }^{* *} P<0.01$ compared with normal control group; ${ }^{\triangle} P<0.05,{ }^{\triangle} P<0.01$ compared with LID group; ${ }^{\#} P<0.05$, ${ }^{\# \#} P<0.01$ compared with normal side.

TABLE 9: Comparison of the relative quantitative mRNA expression of dopamine D1 and D2 receptors in bilateral caudate putamen among different groups.

\begin{tabular}{|c|c|c|c|c|c|}
\hline \multirow{2}{*}{ Group } & \multirow{2}{*}{$n$} & \multicolumn{2}{|c|}{ D1 receptor } & \multicolumn{2}{|c|}{ D2 receptor } \\
\hline & & Lesion side & Normal side & Lesion side & Normal side \\
\hline Normal control & 8 & $1.081 \pm 0.128$ & $0.987 \pm 0.129$ & $1.049 \pm 0.136$ & $1.022 \pm 0.095$ \\
\hline LID & 8 & $1.567 \pm 0.172^{* * \#}$ & $1.363 \pm 0.095^{* *}$ & $0.390 \pm 0.111^{* * \# \#}$ & $0.891 \pm 0.119^{* *}$ \\
\hline TCM & 8 & $1.320 \pm 0.211^{* \Delta \triangle}$ & $1.153 \pm 0.118^{* *}$ & 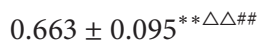 & $1.041 \pm 0.124$ \\
\hline
\end{tabular}

${ }^{*} P<0.05,{ }^{* *} P<0.01$ compared with normal control group; ${ }^{\triangle} P<0.05,{ }^{\triangle} \triangle P<0.01$ compared with LID group; ${ }^{\#} P<0.05$, ${ }^{\# \#} P<0.01$ versus normal side.

group, and the LID group had the biggest drop. The mRNA expression was higher $(P<0.01)$ in the TCM group than that in the LID group. Comparing to the control group, the mRNA expression of normal side showed a significant dip in the LID group $(P<0.01)$, and others had no obvious differences. The expression of lesion side was markedly lower than that of normal side in the LID group and the TCM group $(P<0.01)$.

\subsection{Main Indicators Affecting AIM Score in the Two Groups Mining by ANN}

3.7.1. Relative Importance of Contribution of Input Variables to Output Attribute Values (Difference of AIM Score) in the LID Group. From Table 10, we can see the relative significance of inputs on the output. In the input attribute values, mRNA of D1 lesion side occurred 3 times and the total score was 207.67. And mRNA of D2 lesion side also occurred 3 times and the total score was 171.14. Therefore, in the LID group, the main
TABLE 10: Relative importance of contribution of input variables to output attribute values in LID group (difference of AIM score) in the LID group.

\begin{tabular}{lccc}
\hline Score & $\begin{array}{r}\text { The significance of output attribute values } \\
\text { (difference of AIM score) }\end{array}$ \\
Input attribute values & $<15$ & $15-17.67$ & $17.67-22.47$ \\
\hline mRNA of D1 lesion side & 53.94 & 100 & 53.73 \\
mRNA of D2 lesion side & 38.68 & 66.23 & 66.23 \\
$B_{\max }$ of D1 lesion side & - & - & 100 \\
$B_{\max }$ of D2 lesion side & - & - & - \\
KD of D1 lesion side & 58.41 & - & - \\
KD of D2 lesion side & - & 60.13 & - \\
\hline
\end{tabular}

factors influencing the AIM difference value, in order, were mRNA of D1 lesion side and mRNA of D2 lesion side. 
TABLE 11: Relative importance of contribution of input variables to output attribute values in LID group (difference of AIM score) in the TCM group.

\begin{tabular}{lccccccc}
\hline Score & \multicolumn{7}{c}{ The significance of output attribute values (difference of AIM score) } \\
Input attribute values & -19 & -11 & -10 & -6 & -5 & -2 & 0 \\
\hline mRNA of D1 lesion side & 79.90 & 94.99 & 82.58 & 35.28 & - & 72.79 & - \\
mRNA of D2 lesion side & 99.82 & - & 72.45 & - & - & 73.70 & 100 \\
$B_{\max }$ of D1 lesion side & - & 48.83 & - & 31.13 & 86.70 & - & - \\
$B_{\max }$ of D2 lesion side & 100 & - & - & - & 84.83 & - & - \\
KD of D1 lesion side & - & 33.38 & - & 30.98 & - & - & 87.09 \\
KD of D2 lesion side & - & - & 100 & - & - & 100 & - \\
\hline
\end{tabular}

3.7.2. Relative Importance of Contribution of Input Variables to Output Attribute Values (Difference of AIM Score) in the TCM Group. In the input attribute values, mRNA of D2 lesion side occurred 5 times and the total score was 419.56. And mRNA of D1 lesion side also occurred 5 times and the total score was 365.54. Then, the main factors influencing the AIM difference value, in order, were mRNA of D2 lesion side and mRNA of D1 lesion side (as depicted in Table 11).

\subsection{Main Indicators Affecting Peak Dose Rotation Difference in the Two Groups Mining by ANN}

3.8.1. Relative Importance of Contribution of Input Variables to Output Attribute Values (Difference of Peak Dose Rotation) in the LID Group. As shown in Table 12, in the input attribute values, KD of D2 lesion side, mRNA of D1 lesion side, and mRNA of D2 lesion side occurred 5 times, 3 times, and 3 times, respectively. And the total scores of mRNA of D1 lesion side and mRNA of D2 lesion side are 236.65 and 234.7, respectively. Therefore, in the LID group, the main factors influencing the peak dose rotation difference were KD of D2 lesion side, followed by mRNA of D1 lesion side and mRNA of D2 lesion side.

3.8.2. Relative Importance of Contribution of Input Variables to Output Attribute Values (Difference of Peak Dose Rotation) in the TCM Group. As shown in Table 13, in the input attribute values, mRNA of D2 lesion side, mRNA of D1 lesion side, and $B_{\text {max }}$ of D1 lesion side all occurred 4 times. And the sums of significant scores are $376.54,234.85$, and 180.38 , respectively. Therefore, in the TCM group, the main factors influencing the peak dose rotation difference were mRNA of D2 lesion side, followed by mRNA of D1 lesion side and $B_{\max }$ of D1 lesion side.

\section{Discussion}

At present, the mechanism of LID is not clear yet. Some scholars $[14,15]$ have proposed that LID is due to the abnormal activity of basal ganglia-thalamus-cortex circuit caused by the imbalance between direct pathway (excitability) mediated by dopamine D1 receptor and indirect pathway (suppressant) mediated by dopamine D2 receptor. Some others [16] believed that activation of direct pathway mediated by dopamine D1 receptor might cause LID.
TABLE 12: Relative importance of contribution of input variables to output attribute values (difference of peak dose rotation) in the LID group.

\begin{tabular}{lccccc}
\hline \multirow{2}{*}{ Score } & \multicolumn{5}{c}{ The significance of output attribute values } \\
& \multicolumn{5}{c}{ (peak dose rotation difference) } \\
Input attribute values & 11 & 17 & 19 & 21 & 23 \\
\hline mRNA of D1 lesion side & 100 & 89.80 & - & 46.85 & - \\
mRNA of D2 lesion side & - & 100 & 54.57 & - & 80.13 \\
$B_{\max }$ of D1 lesion side & - & - & 70.56 & - & 86.83 \\
$B_{\max }$ of D2 lesion side & 77.77 & - & - & 74.22 & - \\
KD of D1 lesion side & - & - & - & - & - \\
KD of D2 lesion side & 88.77 & 96.86 & 50 & 100 & 84.66 \\
\hline
\end{tabular}

The results suggested that, with the extension of $\mathrm{LD}$ intraperitoneal injection time, the AIM score and peak dose rotation of rats in the LID model group increased progressively, and the symptoms of LID went worse. At the same time, the LD efficacy time shortened gradually with the LD treatment time prolonged. It was because peripheral pharmacokinetics of LD changed, LD elimination increased, its plasma half-life shortened, and bioavailability decreased, which were consistent with "wearing-off" phenomenon happening to PD patients in clinic [17]. It was found that the activity of dopamine D1 receptor degraded significantly, gene expression was upregulated, and the indirect pathway was inhibited. So we thought that LID is due to not only the activation of direct pathway mediated by dopamine D1 receptor, but also the downregulation (reducing to a certain threshold that induces the overinhibition of indirect pathway) in gene expression and activity of the striatal dopamine D2 receptor caused by LD long-term treatment. The inhibition of basal ganglia indirect pathway, which involves projections from the striatum to GPe and from GPe to the STN, leads to the excitation reduction of projections from STN to GPi and SNr. And the inhibition of projections from GPi and $\mathrm{SNr}$ to thalamus decreasing finally results in the increase in excitation of thalamus and striatum $[18,19]$. When the direct pathway activates, neurotransmitter GABA released by the striatum increased and inhibition to GPi and $\mathrm{SNr}$ improves. The decrease in inhibition to thalamus makes a rise in excitatory neurotransmitter Glu [20]. And it finally caused symptoms of LID [21]. The results also indicated that, 
TABLE 13: Relative importance of contribution of input variables to output attribute values (peak dose rotation difference) in the TCM group.

\begin{tabular}{lcccc}
\hline Score & \multicolumn{4}{c}{ The significance of output attribute values (peak dose rotation difference) } \\
Input attribute values & $-19 \sim-10.67$ & $-10.67 \sim-6.13$ & $-6.13 \sim-1.58$ & 74.55 \\
\hline mRNA of D1 lesion side & 36.07 & 48.12 & 100 & $-1.58 \sim 4.00$ \\
mRNA of D2 lesion side & 100 & 76.54 & 59.65 & 76.11 \\
$B_{\max }$ of D1 lesion side & 24.91 & 32.01 & - & - \\
$B_{\max }$ of D2 lesion side & - & - & - & - \\
KD of D1 lesion side & - & - & - \\
KD of D2 lesion side & - & & - \\
\hline
\end{tabular}

with the extension of LD treatment time, the normal side striatum also had a rise in activity and affinity of D1 receptor, and the gene expression upregulated. And the activity and affinity of D2 receptor declined, and the gene expression downregulated. This illustrated that there might be a bilateral cross dominant phenomenon in dopaminergic neurons of rats. After damage on one side of dopaminergic pathway by 6-OHDA, the cross dominant fiber of contralateral caudate putamen was also damaged.

The artificial neural network is an application similar to the structure of the brain synaptic connections to the mathematical model of information processing. It focuses on extracting the part of the available features to solve the nonlinear problem that computer or other systems cannot solve. Through the analysis of various influence factors, corresponding mathematical model is established to find the best solution by getting the optimal result $[22,23]$. The advantage of ANN is avoiding the model error without hypothesis model of drug behavior [24]. We used BP-ANN to analyze the correlation between neurobehavioral manifestations and activity and gene expression of striatal dopamine D1 and D2 receptors of rats with LID. And we excavated that gene expression of dopamine D1 and D2 receptors was the main factor on AIM score and peak dose rotation of rats with LID and provided a direction for the future research on LID mechanism.

The late Professor Jianhua $\mathrm{Hu}$, Shanghai famous specialist of TCM, extracted Zishenpingchan granule to treat PD on basis of TCM theory, with reference to nonprescription medicine and TCM literature, after decades of exploration and validation. Professor $\mathrm{Hu}$ believed that the pathogenesis of PD was deficiency in origin and excess in superficiality. Deficiency refers to the liver and kidney loss and disorder of viscera function. Excess refers to the collection of wind, fire, phlegm, and blood stasis leading to obturation of brain. So nourishing liver and kidney and unblocking collaterals and relieving toxin were the basic treatment. We confirmed in clinical research that TCM had the function of increasing effect and decreasing toxicity [25]. In previous experimental study, we found Zishenpingchan granule could improve the rotation of $\mathrm{PD}$ rats by eliminating oxygen free radicals [26], increasing the tyrosine hydroxylase in rats and its mRNA expression [27] and restraining dopamine neurons apoptosis [28]. These mechanisms were closely related to LID.

With the extension of LD treatment time, LD efficacy time shortened gradually in the LID group and the change tendency of efficacy time in the TCM group was not obvious, which meant TCM could prolong LD efficacy time, improved motor fluctuations, and extended the duration of "on-time." In the TCM group, the activity of dopamine D1 receptor degraded and gene expression was downregulated and the activity of dopamine D2 receptor increased and gene expression was upregulated, comparing to those in the LID group. The results of ANN showed that TCM lowered AIM score and peak dose rotation of rats by improving the gene expression of dopamine D1 and D2 receptors and reducing the imbalance of receptors. This proved that TCM had multitargets effect. But it significantly improved LID neurobehavioral manifestations indicators mainly through upregulating dopamine D2 receptor gene expression. TCM inhibited the overactivation in direct pathway by decreasing the activity of dopamine D1 receptor and downregulating the gene overexpression. We could find that TCM had a similar effect as D1 receptor blockers. After treatment of TCM, the activity of dopamine D2 receptor rose, gene expression was upregulated, and the inhibition of indirect pathway was reduced. TCM also had a similar effect as D2 receptor agonist. So we thought that TCM prolonged LD efficacy time and effectively relieved LID symptoms through regulating the gene expression and activity of dopamine D1 and D2 receptors, improving the imbalance of direct pathway and indirect pathway and reducing the volatility stimulation of LD on postsynaptic membrane.

\section{Conflict of Interests}

The authors declare that there is no conflict of interests regarding the publication of this paper.

\section{Acknowledgments}

This work was supported by grants from the National Youth Science Project Fund (Grant no. 81302926) and the National Natural Science Foundation of China (Grant no. 30472207).

\section{References}

[1] T. A. Zesiewicz, K. L. Sullivan, and R. A. Hauser, "Levodopainduced dyskinesia in Parkinson's disease: epidemiology, etiology, and treatment," Current Neurology and Neuroscience Reports, vol. 7, no. 4, pp. 302-310, 2007. 
[2] E. V. Encarnacion and R. A. Hauser, "Levodopa-induced dyskinesias in Parkinson's disease: etiology, impact on quality of life, and treatments," European Neurology, vol. 60, no. 2, pp. 57-66, 2008.

[3] X.-B. Cao, Q. Guan, Y. Xu, L. Wang, and S.-G. Sun, "Mechanism of over-activation in direct pathway mediated by dopamine D1 receptor in rats with levodopa-induced dyskinesias," Neuroscience Bulletin, vol. 22, no. 3, pp. 159-164, 2006.

[4] G. Fabbrini, J. M. Brotchie, F. Grandas, M. Nomoto, and C. G. Goetz, "Levodopa-induced dyskinesias," Movement Disorders, vol. 22, no. 10, pp. 1379-1389, 2007.

[5] M. W. Ba, Z. G. Liu, and M. Kong, "Establishment and evaluation of levodopa-induced dyskinesias in Parkinson's disease rats model," Journal of Shanghai Jiaotong University (Medical Science), vol. 26, no. 7, p. 810, 2006.

[6] X. M. Bao and S. Y. Shu, Brain Stereotaxic Atlas of Rats, People's Medical Publishing House, 1991.

[7] L. S. Carman, F. H. Gage, and C. W. Shults, "Partial lesion of the substantia nigra: relation between extent of lesion and rotational behavior," Brain Research, vol. 553, no. 2, pp. 275-283, 1991.

[8] X. B. Cao, S. G. Sun, and Y. Xu, "Experimental research of levodopa-induced dyskinesias in Parkinson's disease," National Medical Journal of China, vol. 84, no. 6, pp. 505-507, 2004.

[9] D. Li, Y. X. Huang, X. J. Huang et al., "Establishment of levodopa-induced dyskinesias in Parkinson's disease rats model by 6-hydroxy dopamine and the evaluation of the behavior," Suzhouuviversity Journal Ofmedical Science, vol. 30, no. 2, pp. 236-239, 2010.

[10] L. Wang, D. Yan, H. B. Xiao et al., "Effects of chitosancoated levodopa nanoliposomes on behaviour and levels of phosphorylated Mr 32000 dopamine- and cyclic adenosine monophosphate-regulated phosphoprotein in rats with dyskinesia," Chinese Journal of Tissue Engineering Research, vol. 16, no. 12, pp. 2125-2128, 2012.

[11] J. Bove, C. Marin, M. Bonastre, and E. Tolosa, "Adenosine A2A antagonism reverses levodopa-induced motor alterations in hemiparkinsonian rats," Synapse, vol. 46, no. 4, pp. 251-257, 2002.

[12] W. M. Zhang, Y. Q. Cai, R. X. Xu et al., "Study the changes of The caudate putamen dopamine D2 receptor activity on rats with Parkinson's disease," Chinese Journal of Neurosurgical Disease Research, vol. 1, no. 3, pp. 266-268, 2002.

[13] M. C. Yan, F. Y. Ye, Y. Q. Zhang, X. Cai, Y. H. Fu, and X. M. Yang, "Optimization model research on efficacy in treatment of chronic urticaria by Chinese and Western Medicine based on a genetic algorithm," Journal of Traditional Chinese Medicine, vol. 33, no. 1, pp. 60-64, 2013.

[14] J. A. Obeso, M. C. Rodriguez-Oroz, M. Rodriguez, M. R. DeLong, and C. W. Olanow, "Pathophysiology of levodopainduced dyskinesias in Parkinson's disease: problems with the current model," Annals of Neurology, vol. 47, no. 4, pp. S22-S34, 2000.

[15] H. Utsumi, Y. Okuma, O. Kano et al., "Evaluation of the efficacy of pramipexole for treating levodopa-induced dyskinesia in patients with Parkinson's disease," Internal Medicine, vol. 52, no. 3, pp. 325-332, 2013.

[16] T. Fieblinger, I. Sebastianutto, C. Alcacer et al., "Mechanisms of dopamine D1 receptor-mediated ERK1/2 activation in the parkinsonian striatum and their modulation by metabotropic glutamate receptor type 5," The Journal of Neuroscience, vol. 34, no. 13, pp. 4728-4740, 2014.
[17] J. M. Brotchie, J. Lee, and K. Venderova, "Levodopa-induced dyskinesia in Parkinson's disease," Journal of Neural Transmission, vol. 112, no. 3, pp. 359-391, 2005.

[18] C. Hyacinthe, Q. Barraud, F. Tison, E. Bezard, and I. Ghorayeb, "D1 receptor agonist improves sleep-wake parameters in experimental parkinsonism," Neurobiology of Disease, vol. 63, pp. 2024, 2014.

[19] L. Frau, M. Morelli, and N. Simola, "Performance of movement in hemiparkinsonian rats influences the modifications induced by dopamine agonists in striatal efferent dynorphinergic neurons," Experimental Neurology, vol. 247, pp. 663-672, 2013.

[20] F. Mela, M. Marti, S. Bido, M. A. Cenci, and M. Morari, "In vivo evidence for a differential contribution of striatal and nigral D1 and D2 receptors to 1-DOPA induced dyskinesia and the accompanying surge of nigral amino acid levels," Neurobiology of Disease, vol. 45, no. 1, pp. 573-582, 2012.

[21] E. Santini, E. Valjent, and G. Fisone, "Parkinson's disease: levodopa-induced dyskinesia and signal transduction," FEBS Journal, vol. 275, no. 7, pp. 1392-1399, 2008.

[22] S. Yamamura, "Clinical application of artificial neural network (ANN) modeling to predict pharmacokinetic parameters of severely ill patients," Advanced Drug Delivery Reviews, vol. 55, no. 9, pp. 1233-1251, 2003.

[23] H. F. Shi and X. M. Yang, "Zhichan decoction on PD after transplantation of neural stem cells in rat brain content of DA and its metabolites related data mining research," in Proceedings of the International Conference of Computation and Evolutionary Computation, vol. 2, pp. 267-270, 2012.

[24] X. M. Yang, L. Y. Xu, F. Zhong, and Y. Zhu, "Data miningbased detection of acupuncture treatment on juvenile myopia," Journal of Traditional Chinese Medicine, vol. 32, no. 3, pp. 372376, 2012.

[25] C. X. Yuan and J. H. Hu, "Clinical observation on nourishing liver and kidney, Tongluo detoxification in tr eating 30 cases of Parkinson's disease," Shanghai Journal of Traditional Chinese Medicine, vol. 38, no. 8, pp. 8-9, 2004.

[26] J. C. He, C. X. Yuan, Y. M. Li et al., "Effect of nourishing liver and kidney, Tongluo detoxification on the oxidative stress of rats with Parkinson's disease," Chinese Journal of New Drugs and Clinical Remedies, vol. 22, no. 3, pp. 160-163, 2003.

[27] J. C. He, C. X. Yuan, and H. C. Wei, "Effect of Madopar Combine with herbal medicine on the Tyrosine hydroxylase (TH) and TH mRNA of rats with Parkinson's disease," Chinese Journal of Gerontology, vol. 26, no. 1, pp. 54-56, 2006.

[28] J. C. He, C. X. Yuan, H. C. Wei et al., "Effect of nourishing liver and kidney, Tongluo detoxification on apoptosis of rats with Parkinson's disease," Chinese Journal of Gerontology, vol. 23, no. 2, pp. 114-116, 2003. 


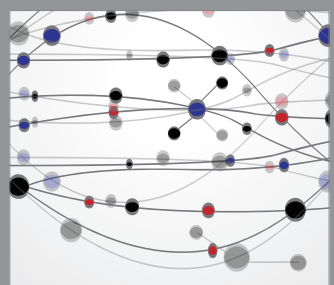

The Scientific World Journal
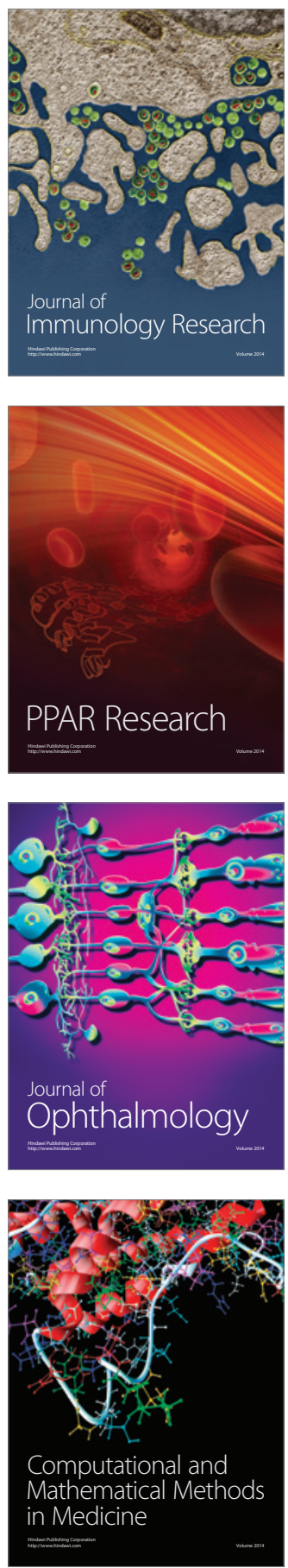

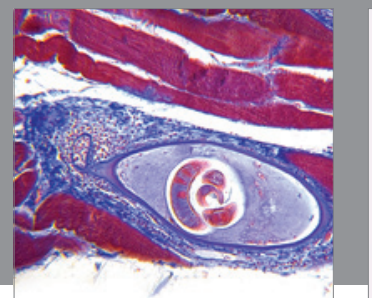

Gastroenterology

Research and Practice
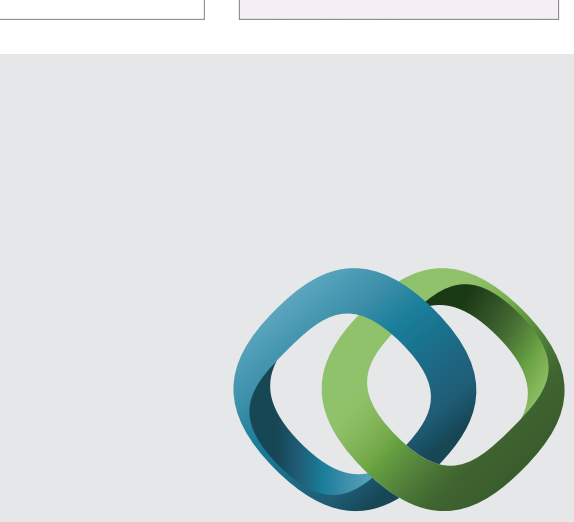

\section{Hindawi}

Submit your manuscripts at

http://www.hindawi.com
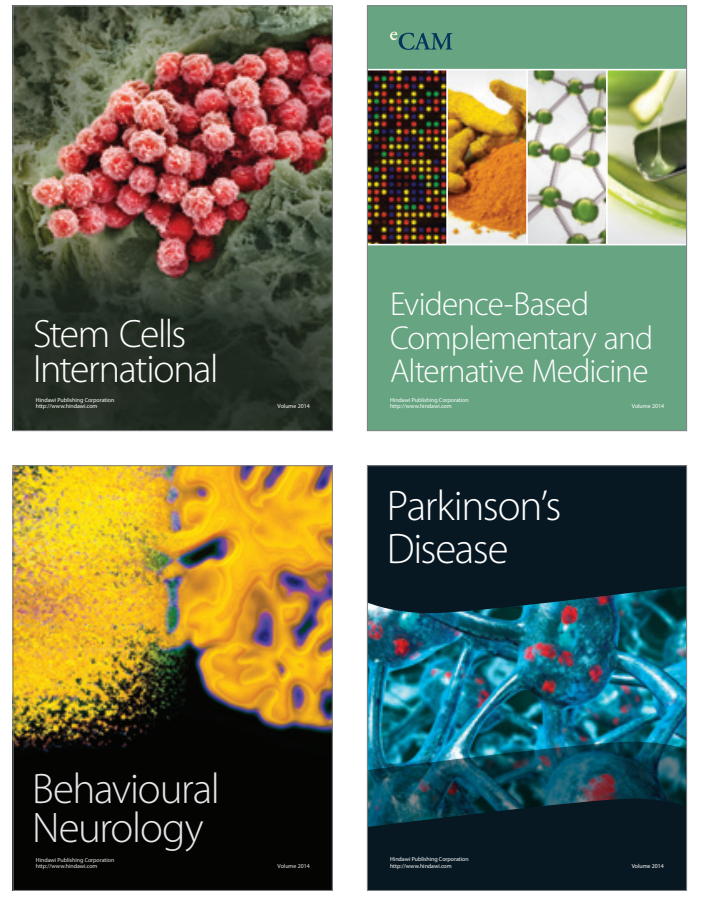
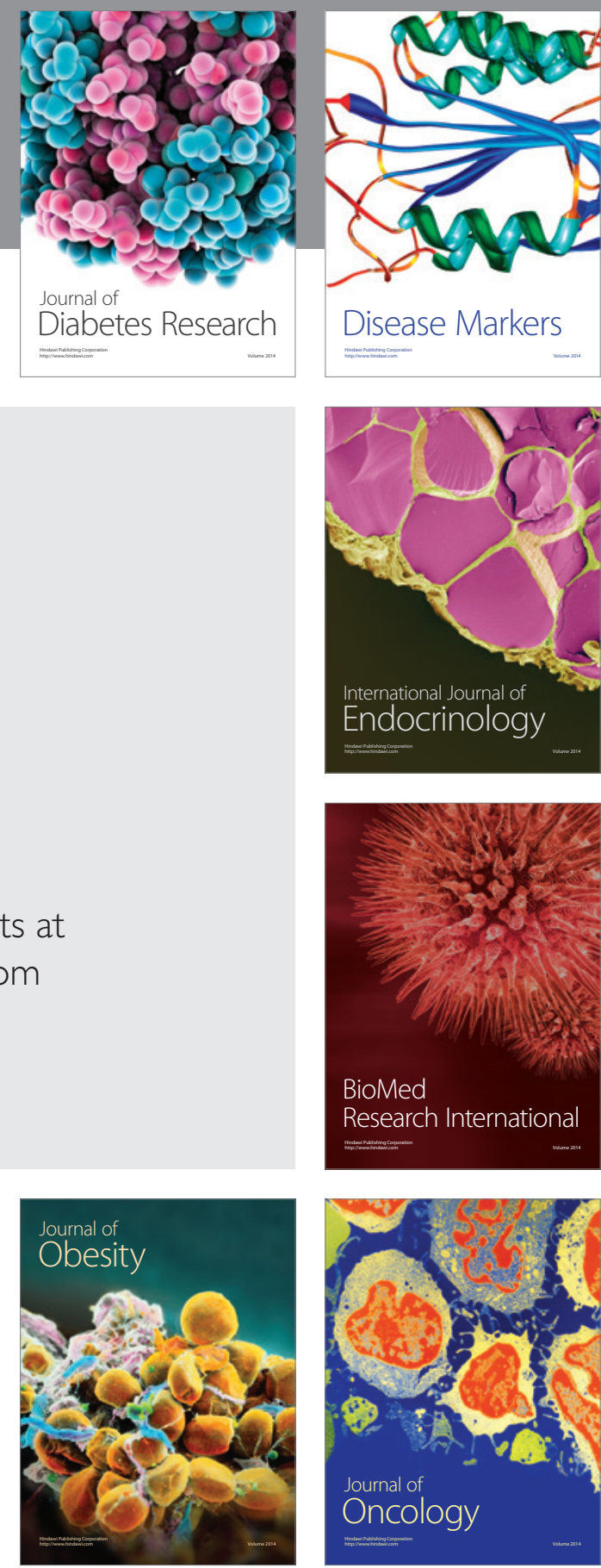

Disease Markers
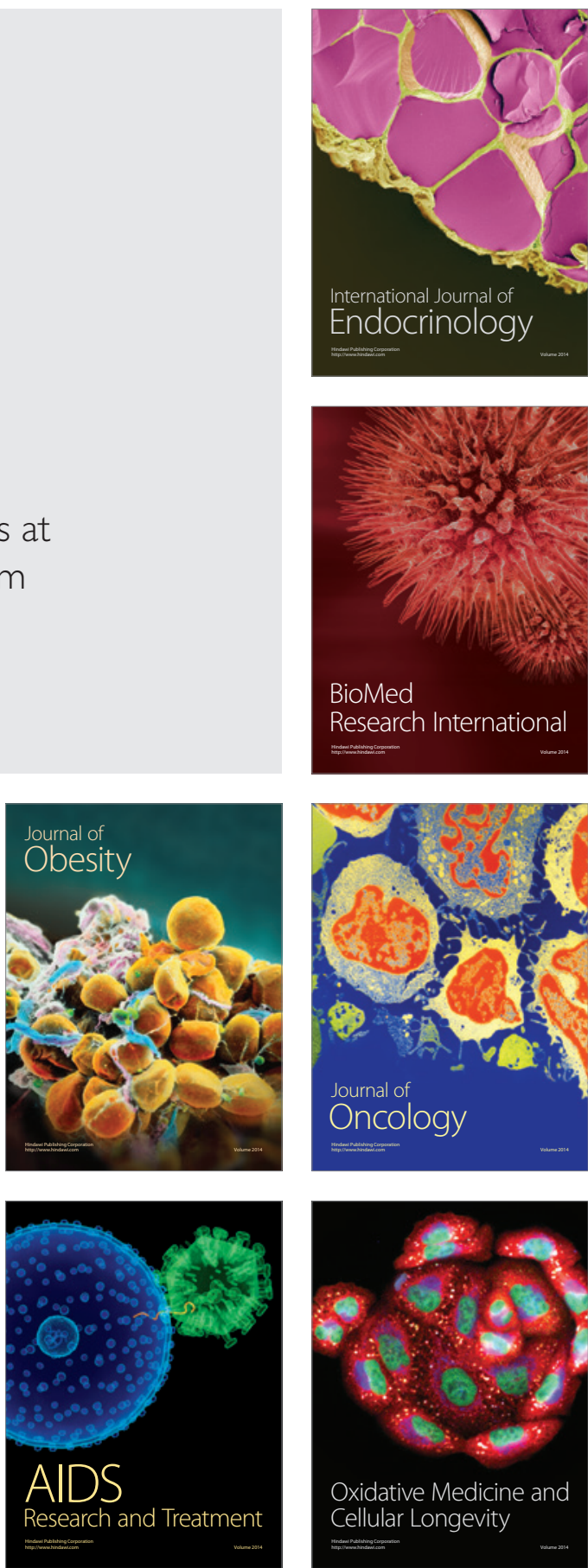